\title{
Cooperative Sensing and Compression in Vehicular Sensor Networks for Urban Monitoring
}

\author{
Xiaoxiao $\mathrm{Yu}^{1}$, Huasha $\mathrm{Zhao}^{1}$, Lin Zhang ${ }^{1}$, Shining $\mathrm{Wu}^{1}$, Bhaskar Krishnamachari ${ }^{2}$, Victor O.K. $\mathrm{Li}^{3}$ \\ ${ }^{1}$ Department of Electronic Engineering, Tsinghua University, Beijing, 100084, China \\ ${ }^{2}$ Viterbi School of Engineering, University of Southern California, USA \\ ${ }^{3}$ Department of Electrical and Electronic Engineering, University of Hong Kong, Hong Kong, China \\ yuxx04@mails.tsinghua.edu.cn
}

\begin{abstract}
A Vehicular Sensor Network (VSN) may be used for urban environment surveillance utilizing vehicle-based sensors to provide an affordable yet good coverage for the urban area. The sensors in VSN enjoy the vehicle's steady power supply and strong computational capacity not available in traditional Wireless Sensor Network (WSN). However, the mobility of the vehicles results in highly dynamic and unpredictable network topology, leading to packet losses and distorted surveillance results. To resolve these problems, we propose a cooperative data sensing and compression approach with zero inter-sensor collaboration overhead based on sparse random projections. The algorithm provides excellent reconstruction accuracy for the sensed field, and by taking advantage of the spatial correlation of the data, enjoys much smaller communication traffic load compared to traditional sampling algorithms in wireless sensor networks. Real urban environment data sets are used in the experiments to test the reconstruction accuracy and energy efficiency under different vehicular mobility models. The results show that our approach is superior to the conventional sampling and interpolation strategy which propagates data in an uncompressed form, with 4-5dB gain in reconstruction quality and $21-55 \%$ savings in communication cost for the same sampling times.
\end{abstract}

\section{INTRODUCTION}

The Wireless Sensor Network (WSN) is a promising technology for urban environment surveillance. However, finegrained environmental data acquisition requires the deployment of a large number of sensor nodes, which is economically infeasible. Recent advances [1][2] in vehicular communications trigger research in using Vehicular Sensor Network (VSN) as an effective and affordable solution for fine-grained urban sensing. Compared to static sensors, vehicular sensor nodes take advantage of their mobility to improve the coverage to the sensed field. However, since the mobile sensors are attached to vehicles, whose mobility may be unpredictable, we may have non-uniform sampling in the monitored area and hence inaccurate data. In addition, vehicular movements change the network topology frequently, making it difficult to transmit large quantity of data sampled by mobile sensors to the aggregator. Therefore, data compression is a must to reduce the overall traffic load for transmission.

This research is supported in part by the National Science Foundation of China (Grant No.60672107), the Hi-tech Research and Development Program of China (Grant No.2006AA10Z261, 2006AA10A301, and 2007AA100408), and the China 973 Project (Grant No.2007CB307105).
A lot of recent work has been focusing on exploiting relaxed precision requirements to compress data and reduce communication load in the network. [3] uses real historical measurements to build a base signal, and then approximate collected data by means of linear projections of the base signal. Guitton et al. [4] study the temporal and spatial correlations among the car-flow data collected, and propose two Fourier-based compression algorithms utilizing single node temporal correlations and spatial correlations separately. These approaches aim to exploit correlations between different time series on a single node or between different spatially distributed nodes.

We present a cooperative data sensing and compression approach, in which urban environment data is considered as an ensemble to be sampled and reconstructed. We examine the temporal and spatial properties of the urban environment field. It is evident that urban environment data ensemble, i.e. urban temperature, air pollution, traffic noise, etc., is highly correlated in the time and space domains [5]. This property is utilized in our approach to improve the compression efficiency and reduce the reconstruction error. The proposed algorithm is based on Compressive Sensing (CS) [6-9], which provides an efficient signal acquisition scheme that exploits the inherent correlations of the signal to reconstruct it from a small collection of random linear projections. While there is prior work [10-12] on static sensor networks, this paper is one of the first to explore compressive sensing in a vehicular/mobile sensor network context.

The merits of our proposed approach are multifold. First, considering the relatively weak computational power of sensors compared with the aggregator, the algorithm is designed to be computationally asymmetric, i.e., easy compression steps in sensor nodes to facilitate the implementation, with the complicated decompression algorithm at the aggregator. Second, sensor nodes execute the compression process independently without extra inter-sensor collaboration overhead, thus reducing the communication traffic significantly. Third, it allows nodes to sample the field independently and randomly, and therefore relaxes the constraint on the movement pattern of sensors, thus providing great flexibility in the deployment of the system. Last and perhaps most important, environment data ensemble is reconstructed with excellent precision at the 
aggregator, when all compressed data is used at the same time on the joint reconstruction. We evaluate the approach on real urban environment data and different vehicular mobility models, and the results show that it achieves higher monitoring accuracy with less overall traffic load, compared with conventional sensing scheme in common use.

The rest of the paper is organized as follows. Section II presents the assumptions of the model, and provides a brief description of the signal property and vehicular mobility patterns. In Section III, we detail our proposed cooperative sensing and compression strategy along with the mathematical analysis. The scheme is evaluated through real data set simulations in Section IV. Finally, we conclude the paper in Section V.

\section{APPLICATION SCENARIO}

In this section, we describe the main assumptions of the application scenario. Given a metropolitan area, a group of cars equipped with environmental sensors roams at will within the area. There is a fixed data aggregator at the center of the area. The cars adopt vehicular short range communication technology that guarantees car-to-car or car-to-aggregator connection within a certain distance. The data are transmitted amongst cars until all the data are transmitted to the aggregator. The network model and vehicular movement patterns will be introduced first. Then we study the signal properties.

We will use the urban heat island monitoring application as an example. Therefore, we assume that taxies equipped with temperature sensors roam around the metropolitan area.

\section{A. Map Model}

We adopt Manhattan Grid Map [13] to model roads and streets of a metropolitan area. The map is basically composed of a number of horizontal and vertical streets. Each street has two lanes in opposite directions. The mobile sensors move along the streets, change their speeds, and choose new directions at intersections with a certain probability.

\section{B. Mobility Model}

Considering the constraints of the city streets, we use two different mobility models to pattern the vehicular movement in the metropolitan area. We will use them in our simulations to evaluate the sampling and compression approach in Section IV.

1) Manhattan Mobility Model (MM) [13]: Assuming that sensors are uniformly distributed in a Manhattan Grid initially, each node has equal chance of choosing any direction from its initial location. A node moves in a chosen direction first and then is switched into a subsequent, randomly selected street when it reaches the next intersection. Suppose that a node has $50 \%$ chance of going straight ahead, $25 \%$ chance of turning right, and $25 \%$ of turning left. The behavior of nodes mimics the movement pattern of cars, which tend to go straight other than turn. In order to eliminate the impact of the edge effect, we assume a node will rejoin the grid from the opposite side when a node moves beyond the grid.
2) City Section Mobility Model (CSM) [14]: The City Section Mobility Model provides realistic movement pattern of taxies in cities since it severely restricts the traveling behavior of mobile nodes. Nodes must follow predefined paths and traffic rules (e.g. speed limits). To start with, each node is randomly put at a point on the grid. Then the node randomly chooses a destination, also represented by a point on the grid. The movement algorithm from the current location to the destination makes a path according to the shortest travelling time between these two points following some driving rules such as speed limit. Upon reaching the destination, the node may stay there for a specified time and then choose a random destination and repeat the process.

\section{Signal Model}

Information about the environment, such as temperature, air pollution, and wind-speed, can be described as a dynamic spatial scalar field. The values and their variations are always scalar properties over a region and fluctuate over time. In this paper, we focus on the temperature, which is a dynamic spatial scalar field defined as a function on a temporal domain and over a spatial field. Formally, we use the following notations for thermal signals. Given a finite two-dimensional spatial set $S$ and a temporal domain $T$, the temperature field ensemble can be denoted by $v(t, x, y) \in V$, where $t \in T,(x, y) \in S$. The temporal domain $T$ and the spatial field $S$ is either continuous or discrete, depending on the application. According to the Manhattan Grid Map model, the spatial field can be divided into $n \times n$ uniform discrete grids. Since temperature of cities varies slowly, we may safely assume that a snapshot of the field can represent the data over a period of time. In this way, we can denote the thermal signals of a city during a certain period of time by a signal matrix,

$$
\mathbf{X}_{2 D}=\left(\begin{array}{ccc}
x_{11} & \cdots & x_{1 n} \\
\vdots & \ddots & \vdots \\
x_{n 1} & \cdots & x_{n n}
\end{array}\right)
$$

This can be written in vector form as follows,

$$
\begin{aligned}
X & =V e c\left(\mathbf{X}_{2 D}\right) \\
& =\left(x_{11}, \cdots, x_{n 1}, x_{12}, \cdots, x_{n 2}, \cdots, x_{1 n}, \cdots, x_{n n}\right)^{T} \\
& =(x(1), x(2), \cdots, x(N))^{T}
\end{aligned}
$$

with $X \in \mathbb{R}^{N}$ and $N=n \times n$. The thermal signals during a certain period of time are expected to exhibit strong spatial correlations. Moreover, the temperature distribution of a city is likely to be sparse in the frequency domain, because, intuitively, the temperature is largely distributed near the average, and the fluctuation is due to local heat sources which are spatially distributed in repeated spatial pattern due to the block-wise structure of the city, and thus result in concentrated distribution in the frequency domain. Therefore, we make the assumption that the temperature field is sparse 


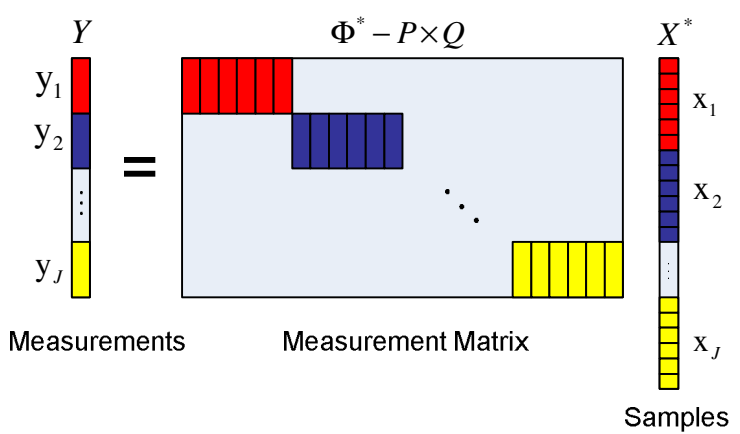

(a) Sampling and Compression

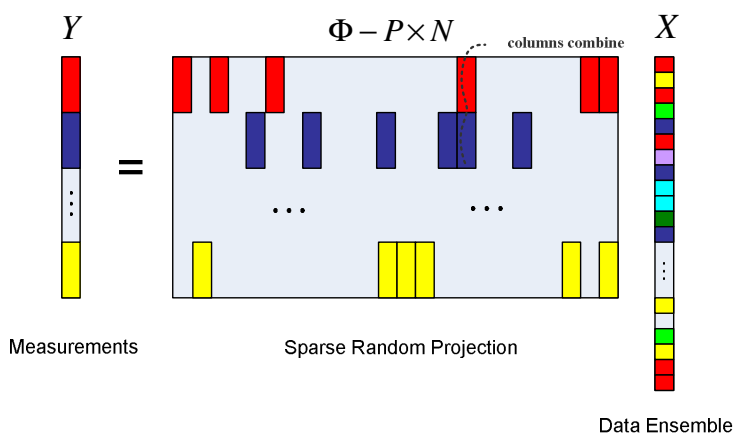

(b) Measurement Matrix Rearrangement

Fig. 1. Illustration of compression scheme based on sparse random projections

in discrete cosine transform (DCT) domain; that is,

$$
X=\Psi \vartheta=\sum_{i=1}^{N} \psi_{i} \vartheta_{i}=\sum_{k=1}^{K} \psi_{k} \vartheta_{k}, \quad \vartheta_{k} \neq 0
$$

where $\Psi$ is the $n \times n$ DCT transform matrix and $\vartheta$ is an $N \times 1$ column vector with $K$ nonzero elements. We say $\vartheta$ is $K$-sparse when $K \ll N$. The assumption is validated based on real urban temperature data analysis in Section IV.

\section{CoOperative SEnSING AND COMPRESSION APPROACH}

We assume that $J$ mobile sensors are deployed in the $n \times n$ metropolitan area to sample the urban thermal data within a certain sampling period. Each vehicular sensor samples several times in this period along its trajectory. Let $\Lambda:=\{1,2, \cdots, J\}$ denote the set of indices for the signals sampled by $J$ sensor nodes. Assuming that, in a certain sampling period, node $j$ makes $M_{j}$ different samples denoted by $\mathrm{x}_{j}=\left(x_{j}(1), x_{j}(2), \cdots, x_{j}\left(M_{j}\right)\right)^{T} \in \mathbb{R}^{M}$, with $x_{j}(i) \in$ $\{x(1), x(2), \cdots, x(N)\}$ and $j \in \Lambda$. We encode $\mathrm{x}_{j}$ to an $L$ dimensional vector $y_{j}$ as,

$$
y_{j}=\Phi_{j} \mathbf{x}_{j}
$$

where $\Phi_{j}$ is as an $L \times M_{j}$ random \pm 1 Bernoulli measurement matrix, containing i.i.d. entries

$$
\phi_{l m}= \begin{cases}+1, & \text { with probablity } \frac{1}{2} \\ -1, & \text { with probablity } \frac{1}{2}\end{cases}
$$

and $1 \leq l \leq L, 1 \leq m \leq M_{j}, L<M_{j}$.

In general, $\Phi_{j}$ is different for each node, and could be generated by a pseudo-random number generator at the nodes or previously allocated to them. In this way, the dimension reduction by random projection is done on mobile nodes in a distributed manner, and only an $L$-dimensional vector is required to be transmitted to the aggregator for signal reconstruction, thus reducing the traffic load. The dimension $L$ of vector $y_{j}$ is a key parameter in the algorithm, and it could affect the amount of traffic load and performance of the data recovery. The impact of $L$ over the system performance will be evaluated by simulation experiments in Section IV.
The data routing algorithm is beyond the scope of this paper, and we simply assume that the aggregator can receive each sensor node's data $y_{j}$ after the sampling period, and then try to recover the thermal field ensemble $X$ of the $n \times n$ metropolitan area. The random measurement matrix $\Phi_{j}$ is generated at each mobile sensor for compressed sensing, and is easily regenerated at the aggregator for signal reconstruction, since we can use the same pseudo-random number generator and synchronize seeds at the sensors and the aggregator. So we have,

$$
Y=\Phi^{*} X^{*}
$$

where $X^{*}=\left[\begin{array}{c}x_{1} \\ x_{2} \\ \vdots \\ x_{J}\end{array}\right], Y=\left[\begin{array}{c}y_{1} \\ y_{2} \\ \vdots \\ y_{J}\end{array}\right], \Phi^{*}=\left[\begin{array}{cccc}\Phi_{1} & 0 & \cdots & 0 \\ 0 & \Phi_{2} & \cdots & 0 \\ \vdots & \vdots & \ddots & \vdots \\ 0 & 0 & \vdots & \Phi_{J}\end{array}\right]$ $\Phi^{*}$ is a $P \times Q$ sparse Bernoulli measurement matrix ( $P=L \times$ $J, Q=\sum_{j=1}^{J} M_{j}$ ) and $X^{*}$ is a $Q$-dimensional vector. For the purpose of recovering the thermal field $X$, we rearrange and combine some columns of $\Phi^{*}$ in accordance with the element index order of $X$, so that,

$$
Y=\Phi X=\Phi \Psi \vartheta
$$

where $X=(x(1), x(2), \cdots, x(N))^{T}$.

Figure 1 demonstrates the compression scheme and matrix rearrangement. Since the paths of different vehicles may overlap and may not cover the whole field, the length of vector $X^{*}$ may be longer or smaller than vector $X$, and the number of columns (say $Q$ ) in $\Phi^{*}$ may not be equal to the number of columns (say $N$ ) in $\Phi$. During the matrix rearrangement processing, several columns of $\Phi^{*}$ would be combined to one column in $\Phi$ when the samples made by different vehicles overlap, as shown in Figure 1(b). The rows, corresponding to the entries of $X$ which are not sampled, would be set to zeros in $\Phi$. Therefore, the final measurement matrix $\Phi$ is a sparse random matrix, which is determined by vehicular movements.

To reconstruct the signal field, the aggregator needs to estimate the $N$-dimensional vector $X$, based on the $P$ dimensional vector $Y$ and the $P \times N$ matrix $\Phi$, where $N \ll P$. The problem can be approximately solved by $L_{1}$ norm minimization algorithms on the conditions that vector $X$ 
has a sparse representation $\vartheta$ under the basis $\Psi$ as described in Section II, and the measurement matrix $\Phi$ satisfies the Uniform Uncertainty Principle (UUP) [8]. The UUP essentially requires that every set of columns of $\Phi$ with cardinality less than $K$ is almost orthogonal. Wang et al.[9] prove that sparse random Bernoulli projections can guarantee reliable recovery, but our measurement matrix is still slightly different: zeros in our measurement matrix are distributed block by block, not randomly distributed as in the sparse projection mentioned in the previous work. Therefore, we conduct numerical experiments to verify our measurement matrix satisfies the reconstruction requirement. We find that the incoherent property of the measurement matrix obtained from the real application scenarios are almost the same with standard sparse random projections at the same sparsity.

\section{Performance Evaluation}

We have evaluated the performance of the sampling and compression approach using real thermal dataset Landsat Thematic Mapper Data (TM) from the U.S. Geological Survey [15]. We choose a snapshot of the temperature distribution of San Jose acquired from TM as the testing data set. This is a $64 \times 64$ thermal signal matrix covering $21.6 \times 21.6$ $\mathrm{Km}$. Figure 2(a) shows the original thermal signal which is rearranged in vector form from the signal matrix, and Figure 2(b) gives its representation in DCT domain. We can see that the representation has only a few large DCT coefficients and many small coefficients. Therefore, it can be regarded as a sparse signal and our assumption is tenable.

The sensing map, the real size of which is $21.6 \times 21.6 \mathrm{Km}$, is rescaled to $64 \times 64$ Manhattan grid. Initially, mobile nodes are deployed uniformly on the grid, and move according to the Manhattan Mobility Model or the City Section Mobility Model mentioned in Section II. We normalize the average velocity of cars to $20 \mathrm{~km} / \mathrm{h}$ or 1.03 minute/grid in the model. The aggregator is placed at the centre of the field.

In our simulation, we use the primal-dual log-barrier algorithm obtained from SparseLab [16] to minimize $L_{1}$-norm and recover the sensing field. Our cooperative compression and reconstruction scheme is compared with the conventional sensing schemes, in which sensors simply sample the sensing field and transmit raw data in an uncompressed form, and
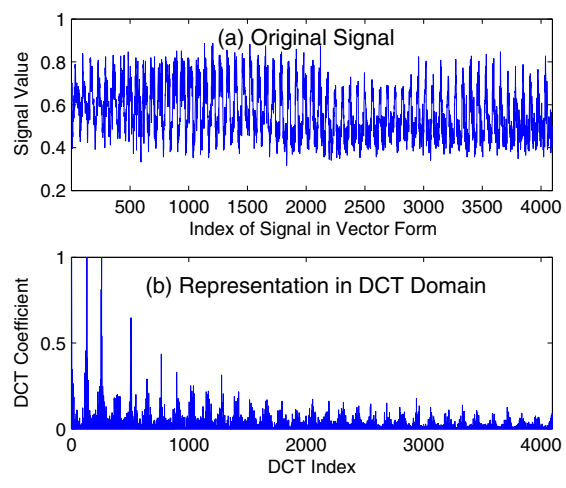

Fig. 4. Temperature Data of San Jose a bilinear interpolation algorithm is used to reconstruct the sensing field. We measure the reconstruction quality and traffic load of the two sensing schemes as we vary i) sensor node mobility model, ii) the number of sensor nodes, and iii) the total sampling period.

Figure 3 shows the impact of number of nodes and total sampling period on the SNR performance. Although more sensor nodes and longer sampling period improves the SNR performance of the two sensing schemes, the two figures consistently show that regardless of the number of nodes, the total sampling period and mobility models, the reconstruction quality of our proposed compression approach is better than that of conventional sensing. Note that our compression and reconstruction approach performs satisfactorily especially at small number of sampling times: when there are only 60 nodes and each node makes 5 samples, our cooperative compression approach is superior to the conventional approach by roughly $8 \mathrm{~dB}$ under both mobility models.

Figure 4 shows the total communication cost of the two sensing schemes. The total communication cost is calculated as $\sum($ Bit $) \times(\text { distance })^{2}$ [19]. We can see that in the two figures plotted with different mobility models, when very few nodes are deployed to sample the field during a short period, total traffic load generated by both the compression scheme and the conventional scheme are similar. But the compression scheme continues to yield significant communication savings compared to the conventional scheme as we increase the number of nodes and total sampling period. Moreover, if we fix the number of nodes and only increase the total sampling period, the communication cost of the compression scheme hardly changes, while in the conventional scheme it rises dramatically.

Finally, we examine the relationship between the recovery quality and total traffic load, when the number of nodes is set to 150 and the total sampling period to 10.3 minutes. Different from Figure 4, we change the value of the parameter $L$ (dimensionality of vector $y_{j}$ ) to reduce the traffic load, though it may also influence the reconstruction accuracy. When given a small error tolerance from $19 \mathrm{~dB}$ to $18 \mathrm{~dB}$ in the reconstruction, our compression strategy yields 39\% traffic load savings. When in the conventional scheme, it would generate 21-55\% more traffic load, but the Avg. SNR is less than $14 \mathrm{~dB}$ by the bilinear interpolation algorithm.

\section{CONClusion}

In this work, we present a cooperative data sensing and compression approach to monitor the urban environment by vehicular sensor networks. Our proposed algorithm is fully distributed and easy to implement in sensor nodes, and the target field is jointly reconstructed with all compressed data at the aggregator. Our novel approach, based on sparse random projections, utilizes vehicles' erratic movements and spatial correlations of urban environment data to achieve significant improvement compared to conventional sensing schemes. Simulation results show that the compression approach offers 21$55 \%$ savings in communication cost, while the reconstruction 


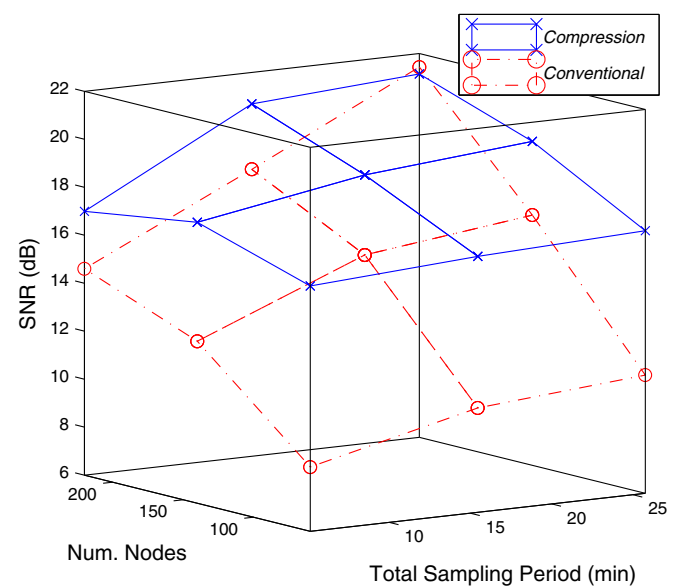

(a) Manhattan Mobility Model

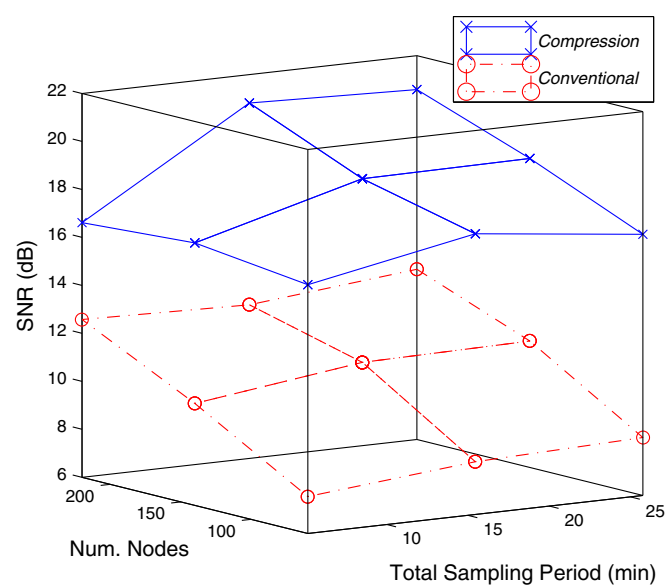

(b) City Section Mobility Model

Fig. 2. Comparison of reconstruction quality between the compression approach and conventional sampling approach using two different mobility models

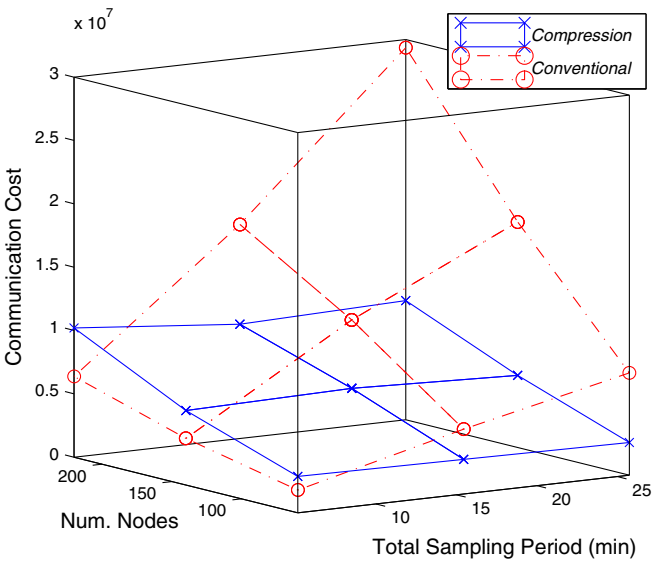

(a) Manhattan Mobility Model

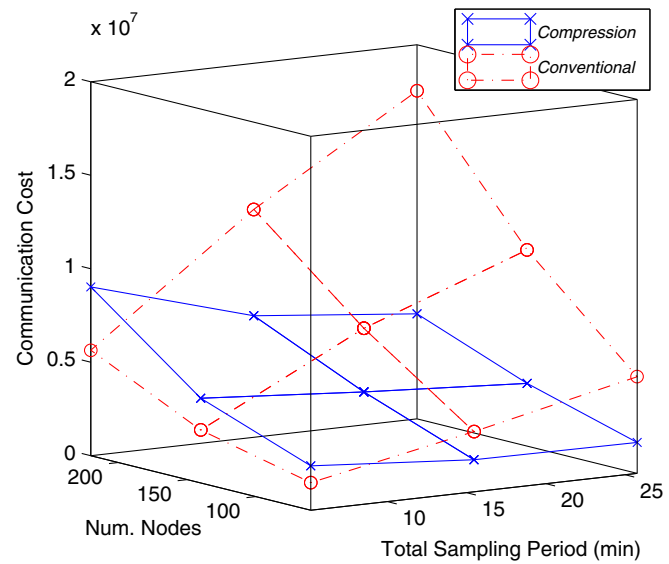

(b) City Section Mobility Model

Fig. 3. Comparison of total communication cost between the compression approach and conventional sampling approach using two different mobility models

quality is improved by $4-5 \mathrm{~dB}$ for the same sampling times. In the future, we plan to exploit the temporal-spatial property of environmental data to optimize sampling and recovery, and investigate how to efficiently transmit real-time data to the aggregator in order to provide accurate and timely information.

\section{REFERENCES}

[1] J. Luo and J.-P. Hubaux, "A Survey of Inter-Vehicle Communication," Technical Report ICTR-200424, EPFL, 2004.

[2] G. Korkmaz, E. Ekici, F. Ozguner and U. Ozguner, "Urban Multi-Hop Broadcast Protocols for Inter-Vehicle Communication Systems," VANET 2004.

[3] A. Deligiannakis, Y. Kotidis and N. Roussopoulos, "Compressing historical information in sensor networks," SIGMOD 2004.

[4] A. Guitton, A. Skordylis and N. Trigoni, "Utilizing Correlations to Compress Time-Series in Traffic Monitoring Sensor Networks," WCNC 2007.

[5] Q. Weng, D. Lu and J. Schubring, "Estimation of land surface temperature-vegetation abundance relationship for urban heat island studies," Remote sensing of environment, vol. 89, no. 4, 2004.

[6] D. Donoho, "Compressed sensing," IEEE Trans. on Information Theory, vol. 52, no. 4, 2006.

[7] E. Candès, J. Romberg and T. Tao, "Robust uncertainty principles: Exact signal reconstruction from highly incomplete frequency information," IEEE Trans. on Information Theory, vol. 52, no. 2, 2006.
[8] E. Candès and T. Tao, "Near optimal signal recovery from random projections: Universal encoding strategies," IEEE Trans. on Information Theory, vol. 52, no. 12, 2006.

[9] W. Wang, M. Garofalakis and K. Ramachandran. "Distributed sparse random projections for refinable approximation," ACM Int. Conf. on Information Processing in Sensor Networks (IPSN), 2007.

[10] S. Lee, S. Pattem, M. Sathiamoorthy, B. Krishnamachari and A. Ortega, "Spatially-Localized Compressed Sensing and Routing in Multi-Hop Sensor Networks," 3rd International Conference on Geosensor Networks, July 2009.

[11] G. Quer, R. Masiero, D. Munaretto, M. Rossi, J. Widmer and M. Zorzi, "On the Interplay Between Routing and Signal Representation for Compressive Sensing in Wireless Sensor Networks," IEEE Information Theory and Applications Workshop, 2009.

[12] J. Meng, H. Li and Z. Han, "Sparse Event Detection in Wireless Sensor Networks using Compressive Sensing," CISS 2009.

[13] F. Bai, N. Sadagopan and A. Helmy, "IMPORTANT: A framework to systematically analyze the Impact of Mobility on Performance of RouTing protocols for Adhoc NeTworks," IEEE INFOCOM 2003.

[14] V. Davies, "Evaluating mobility models within an ad hoc network," Master's thesis, Colorado School of Mines, 2000.

[15] U.S. Geological Survey (USGS), Landsat Thematic Mapper (TM), Available: http://eros.usgs.gov/products/satellite/tm.php

[16] Stanford, SparseLab software package, Available: http://sparselab. stanford.edu/ 